\title{
MULHERES QUE REALIZAM PAPANICOLAOU: CONTRIBUIÇÕES PARA A ESTRATÉGIA SAÚDE DA FAMÍLIA*
}

Helena Hemiko Iwamoto, ${ }^{1}$ Fernanda Carolina Camargo ${ }^{2}$, Marcia Pires de Miranda ${ }^{3}$, Judete Silva Nunes ${ }^{4}$, Isabelle Arruda Barbosa ${ }^{5}$

RESUMO: Estudo epidemiológico, transversal, quantitativo e descritivo com o objetivo de conhecer características sociodemográficas e clínicas das mulheres que realizaram exame de Papanicolaou coletado por enfermeiros da Estratégia Saúde da Família no Município de Novo Cruzeiro, Minas Gerais. Os dados secundários do Sistema de Informação sobre Câncer do Colo de Útero do Departamento de Informática do Ministério da Saúde, do período de 2006 a 2009, foram analisados, totalizando 8.281 exames, na proporção de 0,38 de exames em mulheres de 25 a 59 anos. A maioria estava na faixa etária de 25 a 34 anos (50,54\%), com escolaridade até ensino fundamental $(33,99 \%)$, realizou Papanicolaou anteriormente $(73,92 \%)$ e a última coleta foi entre 1 e 3 anos (61,05\%). Os resultados apontam para a necessidade do fortalecimento das ações educativas e de mobilização comunitária em busca da efetividade da adesão às práticas de cuidado em saúde, no cotidiano do trabalho da Saúde da Família. PALAVRAS-CHAVE: Saúde da mulher; Prevenção de câncer de colo uterino; Epidemiologia; Enfermagem.

\section{WOMEN WHO HAVE TAKEN THE PAPANICOLAOU TEST: CONTRIBUTIONS TO THE 'FAMILY HEALTH' STRATEGY}

ABSTRACT: An epidemiological, transversal, quantitative and descriptive study, with the objective of uncovering the sociodemographic and clinical characteristics of women who had the Papanicolaou test applied by nurses of the Family Health Strategy in the town of Novo Cruzeiro in the Brazilian state of Minas Gerais. Secondary data was assembled from the Ministry of Health's I.T. department's 'System for Information on Cervical Cancer'. The data was from the period 2006 to 2009 and totalled 8,281 exams, in the proportion of 0.38 of exams of women between 25 and 59 years of age. The majority were in the age band 25 to 34 years (50.54\%) educated at least to primary school level (33.99\%), had taken the pap test previously $(73.92 \%)$, and had had the last test within one to three years $(61.05 \%)$. The results point to the need to strengthen educational measures and to mobilize the community in order to increase adhesion to health care practices in the routine $\mathrm{f}$ working with family health.

KEYWORDS: Women's health; Cervix neoplasm prevention; Epidemiology; Nursing.

\section{MUJERES QUE REALIZAN PAPANICOLAOU: CONTRIBUCCIONES PARA LA ESTRATEGIA SALUD DE LA FAMILIA}

RESUMEN: Estudio epidemiológico, transversal, cuantitativo y descriptivo con el objetivo de conocer características sociodemográficas y clínicas de mujeres que realizaran examen de Papanicolaou recogido por enfermeros de la Estrategia Salud de la Familia en el Municipio de Novo Cruzeiro, Minas Gerais. Los datos secundarios del Sistema de Información sobre Cáncer del Cuello de Útero del Departamento de Informática del Ministerio de la Salud, del período de 2006 a 2009, fueron analisados, totalizando 8.281 exámenes, en la proporción de 0,38 exámenes en mujeres de 25 a 59 años. La mayoría estaba en la franja etaria de 25 a 34 años $(50,54 \%)$, con escolaridad hasta enseñanza fundamental (33,99\%), ha realizado Papanicolaou anteriormente $(73,92 \%)$ y la última vez que se recogió los datos fue entre 1 y 3 años $(61,05 \%)$. Los resultados apuntan para la necesidad del fortalecimiento de las acciones educativas y de movilización comunitaria en búsqueda de la efectividad de la adhesión a las prácticas de cuidado en salud, en el cotidiano del trabajo de la Salud de la Familia.

PALABRAS-CLAVE: Salud de la mujer; Prevención de cáncer de cuello uterino; Epidemiología; Enfermería.

\footnotetext{
*Artigo extraído de monografia de conclusão do Curso de Especialização em Atenção Básica em Saúde da Família intitulada "Conhecendo as mulheres que realizam o exame de Papanicolaou na Estratégia Saúde da Família em Novo Cruzeiro/MG” apresentada, em 2010, ao Núcleo de Educação em Saúde Coletiva da Universidade Federal de Minas Gerais - UFMG.
}

${ }^{1}$ Enfermeira. Doutora em Enfermagem Fundamental. Professora da Universidade Federal do Triângulo Mineiro - UFTM.

${ }^{2}$ Enfermeira. Mestre em Atenção à Saúde. Tutora do Curso de Especialização em Atenção Básica em Saúde da Família do Núcleo de Educação em Saúde Coletiva da UFMG.

${ }^{3}$ Enfermeira. Especialista em Atenção Básicae Saúde da Família. Coordenadora da Atenção Básica da Secretaria Municipal de Saúde de Novo Cruzeiro-MG. ${ }^{4}$ Enfermeira. Especialista em Atenção BásicaeSaúde daFamília. Enfermeira da Estratégia Saúde daFamília da Secretaria Municipal deSaúde deUberaba-MG. ${ }^{5}$ Enfermeira. Especialista em Atenção Básica e Saúde da Família. Enfermeira da Estratégia Saúde da Família da Secretaria Municipal de Saúde de Pedras Maria da Cruz-MG. 


\section{INTRODUÇÃO}

$\mathrm{Na}$ atualidade, mesmo diante dos avanços tecnológicos em saúde, o câncer de colo de útero continua se mantendo como um grave problema de saúde pública devido sua elevada morbimortalidade. No Brasil, ele é considerado o tumor mais incidente na Região Norte, o segundo nas Regiões Sul, Centro Oeste e Nordeste e o terceiro na Região Sudeste ${ }^{(1-3)}$.

Estudos ressaltam que os fatores de risco para o câncer de colo de útero são: início precoce de atividade sexual, multiplicidade de parceiros sexuais, desnutrição, tabagismo, infecção pelo HPV (Vírus do Papiloma Humano), baixas condições socioeconômicas, déficit de higiene, uso prolongado de contraceptivos orais e história de infecções sexualmente transmitidas ${ }^{(1-4)}$.

Nas últimas décadas, na medida em que se ampliaram os conhecimentos sobre os fatores de risco, houve crescimento das possibilidades de inserção das mulheres nos programas de prevenção e controle desse agravo. Adiciona-se, como fator de adesão para o controle, o melhor acesso aos serviços de saúde, a captação do público-alvo em ações estratégicas e a capacitação dos profissionais que prestam assistência às mulheres ${ }^{(5-9)}$.

Contudo, as características do desenvolvimento do câncer de colo de útero - lento e silencioso - associadas aos comportamentos da falta de regularidade de busca aos serviços de saúde pelas mulheres, fazem com que este diagnóstico seja realizado tardiamente, quando as possibilidades terapêuticas e a sobrevida já não são animadoras. A detecção precoce do câncer de colo de útero (CCU) tem caráter preventivo ao se considerar as possibilidades de intervenção terapêutica já no nível secundário de atenção, capaz de conter a evolução de possíveis lesões malignas ${ }^{(2,6)}$.

O Ministério da Saúde (MS) preconiza, como estratégia prioritária de prevenção deste agravo, em mulheres de 25 a 59 anos, a realização do esfregaço vaginal - o exame de Papanicolaou - e a implementação desta prática deve ocorrer no cotidiano de trabalho das equipes da Atenção Primária em Saúde (APS), através da Estratégia Saúde da Família (ESF) ${ }^{(1,3,7-11)}$.

Estudos apontam que em torno de $40 \%$ da população feminina brasileira nunca foi submetida a esse exame, e que apenas $7,7 \%$ das mulheres, público-alvo dessa ação, têm a cobertura efetiva de programas governamentais ${ }^{(11)}$. Portanto, é preocupante olhar para a atual disponibilidade dos serviços de saúde em APS, e observar que muitos funcionam de forma fragmentada, contribuindo para o perpetuamento de atitudes que se distanciam da prática de prevenção e promoção da saúde ${ }^{(8-11)}$.
Por mais que as ações de prevenção de CCU tenham aumentado nos últimos anos, ainda há uma elevada tendência de mortalidade por esse $\operatorname{agravo}^{(2,6,12)}$. Alguns estudos têm detectado que mulheres com diagnóstico de CCU nunca realizaram o exame Papanicola$\mathrm{ou}^{(6,12)}$. Por outro lado, os serviços de saúde ainda estão mais voltados para o controle de agravos imediatos, evidenciados na queixa da clientela, afastando-se da atenção integral quando limitam a incorporação de práticas preventivas no cotidiano do trabalho ${ }^{(2,6)}$.

Por essas razões, evidencia-se a necessidade de realização de pesquisas a respeito do tema, para sua compreensão, e fundamentação de propostas de intervenções efetivas, considerando o processo de trabalho das equipes de saúde e a realidade social da mulher brasileira. Assim, esse estudo teve por objetivo descrever características sociodemográficas e clínicas das mulheres que realizaram o exame de Papanicolaou nas equipes da Estratégia Saúde da Família do Município mineiro de Novo Cruzeiro.

\section{METODOLOGIA}

Trata-se de estudo epidemiológico, transversal ${ }^{(13)}$, quantitativo, descritivo, tendo como população mulheres com características sociodemográficas e clínicas que realizaram exame de Papanicolaou, em Novo Cruzeiro-MG.

O Município de Novo Cruzeiro está localizado no noroeste do Estado de Minas Gerais, com 31.319 habitantes, de acordo com projeções intercensitárias para $2009^{(14)}$. A maior parte da população concentra-se na faixa etária economicamente produtiva, entre 15 a 59 anos (54,91\%), e apresenta uma proporção de 101 homens para cada 100 mulheres, esta proporção começou a se diferenciar a partir dos 50 anos, atingindo uma proporção de 72 idosos para cada 100 idosas.

A obtenção dos dados sobre as características sociodemográficas das mulheres que realizaram Papanicolaou foi através do sítio eletrônico do Departamento de Informática do Sistema Único de Saúde (DATASUS), pelo Sistema de Informação do Câncer do Colo do Útero (SISCOLO) versão 4.0, para o período de 2006 a $2009^{(14)}$. A análise foi realizada por estatística descritiva através de frequências absolutas (n) e em porcentagem (\%), por medidas de dispersão como média, desvio padrão (S) e o coeficiente de variação (CV), considerando elevado para aqueles acima de $25 \%$.

Foi considerado o parâmetro proposto pelo Plano Diretor da Atenção Primária à Saúde do Estado de Minas Gerais (PDAPS) que prevê um dimensionamento 
quantitativo sobre as mulheres da faixa etária de 25 a 59 anos, de cobertura de $33 \%$ ao ano, quanto à realização do exame de Papanicolaou ${ }^{(15)}$. Todas as informações coletadas foram armazenadas em banco de dados construído no software Excel $^{\mathbb{R}}$, e os cálculos propostos também se deram por meio desse operador. O trabalho desenvolveuse de modo a garantir o cumprimento dos preceitos da Resolução 196/96 do Conselho Nacional de Saúde.

\section{RESULTADOS}

Conforme registros do SISCOLO/DATASUS foram realizados 8.281 exames de Papanicolaou pela ESF em Novo Cruzeiro-MG, no período de 2006 a 2009, em sua maioria coletados por enfermeiros. No decorrer desse período, houve aumento gradativo de realização desses exames, passando de 1.741 em 2006, para 1.849 em 2007 e 1.951 em 2008. O maior número de exames realizados foi em 2009, 2.740 exames. Para o período e faixa-etária estudados era esperada a realização de 5.463 exames de Papanicolaou. Entretanto, foram realizados 6.353 exames, com uma média de 1.588 exames/ano [ $\mathrm{S}=$ 322,20 e $\mathrm{CV}=20,91 \%$ ] (Tabela 1).

Tabela 1- Distribuição da cobertura de exames de Papanicolaou realizados em mulheres de 25 a 59 anos. Novo Cruzeiro, 2006 a 2009

\begin{tabular}{lccccc}
\hline Ano & $\mathbf{2 0 0 6}$ & $\mathbf{2 0 0 7}$ & $\mathbf{2 0 0 8}$ & $\mathbf{2 0 0 9}$ & Total \\
\hline Esperados & 1.304 & 1.332 & 1402 & 1426 & 5.463 \\
Realizados & 1.368 & 1.423 & 1.497 & 2065 & 6.353 \\
Cobertura & $105 \%$ & $107 \%$ & $107 \%$ & $145 \%$ & $116 \%$ \\
\hline
\end{tabular}

De acordo com o PDAPS, a proporção entre as mulheres da faixa etária de 25 a 59 anos que realizaram Papanicolaou, anualmente, sobre todas as mulheres da mesma faixa etária e período, apresentou uma evolução ascendente. Os valores dessa proporção foram de 0,35 para os três primeiros anos e de 0,48 para 2009. Dessa forma, a média dessa proporção foi de 0,38 [S=0,06 e CV=16,7\%].

A média da idade das mulheres que realizaram exames foi de 30 anos [S=13,25; $\mathrm{CV}=35,8 \%]$, com maior concentração entre 25 a 34 anos $(27,2 \%)$ e 35 a 44 anos (23,34\%). Os exames realizados em mulheres com 60 anos e mais perfizeram 8,32\%, enquanto os realizados em menores de 19 anos foram 4,65\%. Em relação à escolaridade, a soma numérica das mulheres com ensino fundamental incompleto e analfabetas foi a maioria (30,23\%). Ressalta-se a baixa qualidade desta informação na fonte de dados pesquisada, uma vez que mais da metade dos registros (61,89\%) encontravam-se como "ignorada", como apresentado na tabela 2(p. 427).
Do total de mulheres que realizaram o exame de Papanicolaou, $73,92 \%$ já tinham realizado anteriormente, com média anual de 1.530 [S=363,3; CV=23,7\%], 20,15\% das mulheres nunca haviam realizado o exame, com média anual de 417 [ $S=43,7 ; C V=10,5 \%]$, e 4,3\%, não souberam informar se já tinha feito o exame anteriormente (Tabela 2).

Quanto ao espaço de tempo entre a realização do exame de Papanicolaou, $35,70 \%$ das mulheres afirmaram que realizaram o último exame há um ano, 19,2\% há dois anos, $16,15 \%$ há três anos, $4,71 \%$ tinham feito o exame há mais de cinco anos, e em torno de $4 \%$ das mulheres fizeram o exame no mesmo ano (Tabela 2).

\section{DISCUSSÃO}

Em Novo Cruzeiro, o modelo assistencial de organização da APS apresenta, como forma preferencial de trabalho, a ESF, com cobertura de aproximadamente $100 \%$ da população residente. Em relação à saúde suplementar, em 2009, somente 355 pessoas declararam possuir convênios para assistência médica, revelando com isso que a maioria da população residente é SUS dependente ${ }^{(14-15)}$. Uma das maiores causas de mortalidade no município são as neoplasias. Em 2006, a mortalidade por neoplasia maligna de colo uterino foi de 6,6 mulheres por 100 mil habitantes, enquanto para esse mesmo período, em Minas Gerais e no Brasil, a mortalidade foi de 5,4 e 4,8/100.000, respectivamente ${ }^{(14)}$.

Para obtenção de impacto epidemiológico na frequência da distribuição do CCU, a Organização Mundial de Saúde estabelece como necessária a realização do exame de Papanicolaou que atinja uma cobertura de $80 \%$ da população feminina ${ }^{(16)}$. Neste sentido, vale destacar os esforços dos enfermeiros para o alcance desta cobertura, principalmente, nas regiões Sul e Sudeste do Brasil ${ }^{(4,10)}$.

Estudo realizado em Londrina-PR demonstrou que das 513 mulheres cadastradas na ESF, 53,8\% fizeram o exame de Papanicolaou anualmente, $26,9 \%$ de um a três anos e $8 \%$ nunca o fizeram ${ }^{(17)}$. Em São Leopoldo-RS, das 867 mulheres residentes na zona urbana, $85,5 \%$ já tinham realizado o exame nos últimos três anos e 7,6\% das mulheres nunca tinham feito o exame ${ }^{(18)}$. Já na periferia do Distrito Oeste, em Natal-RN, estudo realizado com 120 mulheres de uma UBS constatou que $60 \%$ delas realizaram o exame anualmente, $58 \%$ consideraram a realização do exame e o tratamento adequados, e apenas $17 \%$ acreditavam que o exame tinha a finalidade de identificar alterações uterinas ${ }^{(19)}$. 
Em Pernambuco, o estudo realizado com 258 mulheres, de 18 a 69 anos, constatou que $82 \%$ daquelas que tinham realizado o exame de Papanicolaou, nos últimos três anos, tinham de 25 a 59 anos, sendo mais prevalente entre as mulheres com ensino fundamental completo; $63,6 \%$ delas frequentavam o serviço de saúde regularmente ${ }^{(11)}$. Quanto ao conhecimento sobre a importância da realização do exame, das 476 mulheres estudantes do ensino médio noturno de escolas públicas de Uberaba-MG, de 18 a 65 anos, 96\% responderam corretamente sobre a periodicidade de realização do exame, $85 \%$ conheciam a sua finalidade e $96 \%$ sabiam que o exame é feito em Unidades Básicas de Saúde ${ }^{(9)}$.

A realização do exame de Papanicolaou é mais eficaz em países desenvolvidos como Itália e Inglaterra ${ }^{(1,4)}$, onde a prevalência de mulheres que realizaram o exame, pelo menos uma vez na vida, foi de $53 \%$ entre italianas, e de $77 \%$ entre inglesas. Nos países latinos, a prevalências de mulheres que realizaram o Papanicolaou, pelo menos uma vez na vida, foi mais baixa, em torno de $30 \%{ }^{(4)}$.

Estudos apontam como fatores que levam as mulheres à não-realização do exame, principalmente aqueles relativos ao baixo nível socioeconômico, pouca escolaridade, renda familiar precária e o fato de pertencer às faixas etárias mais jovens ${ }^{(3,5,10)}$. As iniquidades sociais, mesmo em áreas de cobertura de ESF, são evidenciadas pela baixa adesão para a realização do Papanicolaou entre mulheres de classes econômicas menos favorecidas, não brancas e com menor escolaridade ${ }^{(6-7,18)}$.

Quanto aos motivos que levam as mulheres a realizar regularmente o exame, em países em desenvolvimento como Brasil, tem-se: o conhecimento das atividades programadas pelas UBS, a proximidade dessas às residências, recomendações médicas e as crenças individuais em saúde ${ }^{(4,17-19)}$. De certa forma, há uma associação entre proximidade da unidade de saúde, vínculo com equipe e frequência na realização do exame ${ }^{(17,20)}$.

A prevenção do CCU é de baixo custo, ao se considerar o custo das consequências desse agravo. E, a solução para o seu enfrentamento está diretamente relacionada com o compromisso dos profissionais de saúde, incentivando as práticas de proteção em saúde; com os gestores em saúde, formulando e implementando políticas públicas; e com a própria população, fortalecendo sua participação social nesse processo ${ }^{(1,16,20)}$.

Baseado nessas informações, é necessário que o enfermeiro contribua para o fortalecimento da reorganização dos serviços de saúde, em especial a APS/ESF, na busca de maior adesão das mulheres às práticas de promoção da saúde. Vale destacar que, para o reconhecimento desses fatores, no cotidiano de trabalho das equipes de saúde, evidencia-se a necessidade de mudanças na organização do Sistema de Saúde brasileiro. Essas mudanças

Tabela 2 - Caracterização sociodemográfica e clínica das mulheres que realizaram o exame de Papanicolaou. Novo Cruzeiro, 2006 a 2009

\begin{tabular}{|c|c|c|c|c|c|c|c|}
\hline \multicolumn{8}{|c|}{ Realização anterior de exame de Papanicolaou } \\
\hline Nunca realizou & \multicolumn{2}{|c|}{ Não sabe se já realizou } & \multicolumn{2}{|c|}{ Refere já ter realizado } & Não informou & Ignorado & Total \\
\hline $1669(20,15 \%)$ & \multicolumn{2}{|c|}{$346(4,18 \%)$} & \multicolumn{2}{|c|}{$6121(73,92 \%)$} & $10(0,12 \%)$ & $117(1,63 \%)$ & $8281(100 \%)$ \\
\hline \multicolumn{8}{|c|}{ Tempo de coleta após a realização do último exame } \\
\hline $\begin{array}{c}\text { Mesmo ano } \\
262 \\
(3,16 \%)\end{array}$ & $\begin{array}{c}1 \text { ano } \\
2.957 \\
(35,70 \%)\end{array}$ & $\begin{array}{c}2 \text { anos } \\
1.590 \\
(19,20 \%)\end{array}$ & $\begin{array}{c}3 \text { anos } \\
510 \\
(6,15 \%)\end{array}$ & $\begin{array}{c}4 \text { anos } \\
206 \\
(2,48 \%)\end{array}$ & $\begin{array}{c}\text { 5anos } \\
390 \\
(4,71 \%)\end{array}$ & $\begin{array}{c}\text { Ignorado } \\
2366 \\
(28,6 \%)\end{array}$ & $\begin{array}{c}\text { Total } \\
8281 \\
(100 \%)\end{array}$ \\
\hline \multicolumn{8}{|c|}{ Faixa etária (anos) } \\
\hline $\begin{array}{c}<25 \\
1239 \\
(14,96 \%)\end{array}$ & $\begin{array}{c}25 \mathrm{-}-35 \\
2253 \\
(27,21 \%)\end{array}$ & $\begin{array}{c}35 \mid-45 \\
1933 \\
(23,34 \%)\end{array}$ & $\begin{array}{c}45 \mid-55 \\
1609 \\
(19,43 \%)\end{array}$ & $\begin{array}{c}55 \mid-65 \\
855 \\
(10,32 \%)\end{array}$ & $\begin{array}{c}>65 \\
392 \\
(4,73 \%)\end{array}$ & \multicolumn{2}{|c|}{$\begin{array}{l}\text { Total } \\
8281 \\
(100 \%)\end{array}$} \\
\hline \multicolumn{8}{|l|}{ Escolaridade } \\
\hline Analfabeta & \multicolumn{2}{|c|}{$\begin{array}{l}\text { Fundamental } \\
\text { incompleto }\end{array}$} & $\begin{array}{l}\text { Fundamental } \\
\text { completo }\end{array}$ & $\begin{array}{l}\text { Médio } \\
\text { completo }\end{array}$ & $\begin{array}{l}\text { Superior } \\
\text { completo }\end{array}$ & Ignorado & Total \\
\hline $\begin{array}{c}789 \\
(9,53 \%)\end{array}$ & \multicolumn{2}{|c|}{$\begin{array}{c}1714 \\
(20,70 \%)\end{array}$} & $\begin{array}{c}311 \\
(3,76 \%)\end{array}$ & $\begin{array}{c}267 \\
(3,22 \%)\end{array}$ & $\begin{array}{c}75 \\
(0,91 \%)\end{array}$ & $\begin{array}{c}5125 \\
(61,89 \%)\end{array}$ & $\begin{array}{c}8281 \\
(100 \%)\end{array}$ \\
\hline
\end{tabular}


relacionam-se à operacionalização de critérios como efetividade, otimização e acesso, em que tecnologias sofisticadas de altíssimo custo possam ser substituídas por aquelas que considerem intervenções mais resolutivas, quando se trata do agravo $\mathrm{CCU}^{(3,5-7,20)}$.

Em contrapartida, destaca-se a sensibilidade do enfermeiro para a compreensão dos fatores que envolvem a prevenção do CCU, como as subjetividades do corpo feminino, seus aspectos psicoafetivos e as relações que essas mulheres estabelecem no seu modo de ser e estar na vida ${ }^{(6,9,12,20)}$.

Entre as ações indicadas, incluem-se identificar, na busca ativa, das mulheres faltosas às consultas, fatores como: dificuldades de acesso, problemas domésticos, motivos conjugais, desinformação sobre o exame, ausência de problemas ginecológicos, sentimentos de embaraço, vergonha ou desconforto físico, problemas na relação entre equipe de saúde e a mulher, medo em relação ao câncer e ao próprio exame ${ }^{(4,8,18)}$.

A prevenção e controle do CCU continuam como relevante questão de saúde pública ao se considerar a alta taxa de mortalidade por esse agravo, a probabilidade de cura ao ser diagnosticado precocemente e a possibilidade de prevenção, por meio da educação em saúde. Nesse sentido, é importante identificar a demanda que busca as ações de diagnóstico precoce e prevenção desse agravo, e assim, reorganizar os serviços de saúde para contemplar integralmente a população feminina que necessita dessa assistência.

\section{CONCLUSÃO}

No período de 2006 a 2009, o número de exames de Papanicolaou realizados no Município de Novo CruzeiroMG foi maior que o número esperado. A média de idade para realização do exame foi 30 anos, com predomínio na faixa etária de 25 a 44 anos. Quanto à escolaridade houve predomínio do ensino fundamental incompleto e de analfabetas. A maioria das mulheres já tinha realizado o exame alguma vez na vida, e o período de realização da coleta foi de 1 a 3 anos.

De forma geral, os dados do SISCOLO apresentaram-se como informações de extrema utilidade para a organização da assistência à saúde da mulher na APS/ ESF. Esta investigação aponta para a necessidade imprescindível do enfermeiro fortalecer as ações educativas e de mobilização comunitária, para conscientização permanente do público-alvo e adesão ininterrupta das mulheres nas estratégias de prevenção do CCU.

É importante promover mudanças de comportamento da população assistida, afim de que haja maior adesão ao exame de Papanicolaou, para investigação e controle cada vez mais efetivo do CCU. Portanto, é necessária a adoção de proposições inovadoras no cotidiano assistencial, que considerem a busca ativa das mulheres-alvo e intervenções nos domicílios ou em espaços comunitários. Enfim, cabe aos enfermeiros e às equipes de ESF o planejamento de ações em saúde que promovam o fortalecimento do processo de trabalho frente à necessidade do diagnóstico precoce do $\mathrm{CCU}$, e com isso garantir o melhor cuidado à população feminina.

\section{REFERÊNCIAS}

1. Ministério da Saúde (BR). Secretaria de Atenção à Saúde. Departamento de Atenção Básica. Cadernos de Atenção Básica. Controle dos cânceres do colo do útero e de mama. Brasília: Ministério da Saúde; 2006.

2. Wolschick NM, Consolaro MEL, Suzuki LE, Boer CG. Câncer do colo do útero: tecnologias emergentes no diagnóstico, tratamento e prevenção da doença. Rev Bras Anal Clin. 2007;39(2):123-9.

3. Greenwood SA, Machado MFAS, Sampaio NMV. Motivos que levam mulheres a não retornarem para receber o resultado de exame Papanicolaou. Rev LatinoAm Enfermagem. 2006;14(4):503-9.

4. Pinho AA, França-Junior I. Prevenção do câncer de colo do útero: um modelo teórico para analisar o acesso e a utilização do teste de Papanicolaou. Rev Bras Saude Mater Infant. 2003;3(1):95-112.

5. Soares MC, Mishima SM, Meincke SMK, Simino GPR. Câncer de colo uterino: caracterização das mulheres em um município do Sul do Brasil. Esc Anna Nery Rev Enferm. 2010;14(1):90-6.

6. Cirino FMSB, Nichiata LYI, Borges ALV. Conhecimento, atitudes e práticas na prevenção do câncer de colo uterino e HPV em adolescentes. Esc Anna Nery. 2010;14(1):126-34.

7. Fernandes LCL, Bertoldi AD, Barros AJD. Utilização dos serviços de saúde pela população coberta pela estratégia de saúde da família. Rev Saude Publica. 2009;43(4):595-603.

8. Oliveira MM, Pinto IC. Percepção das usuárias sobre as ações de prevenção do câncer do colo do útero na estratégia saúde da família em uma distrital de saúde do município de Ribeirão Preto, São Paulo, Brasil. Rev Bras Saude Mater Infant. 2007;7(1):31-8.

9. Valente CA, Andrade V, Soares MBO, Silva SR. 
Conhecimento de mulheres sobre o exame de Papanicolaou. Rev Esc Enferm USP. 2009;43(n. esp 2):1193-8.

10. Martins LFL, Thuler LCS, Valente GC. Cobertura do exame de Papanicolaou no Brasil e seus fatores determinantes: uma revisão sistemática da literatura. Rev Bras Ginecol Obstet. 2005;27(8):485-92.

11. Albuquerque KM, Frias PG, Andrade CLT, Aquino EML, Menezes G, Swarcwald CL. Cobertura do teste de Papanicolaou e fatores associados à não-realização: um olhar sobre o programa de prevenção do câncer do colo do útero em Pernambuco, Brasil. Cad Saude Publica. 2009;25 Supl 2:S301-9.

12. Eduardo KGT, Ferreira ERM, Pinheiro AKB, Ximenes LB. Utilização do instrumento de melhoria de desempenho na avaliação do exame de Papanicolaou por enfermeiros. Cogitare Enferm. 2008;13(3):329-35.

13. Almeida Filho N, Rouquayrol MZ. Elementos de metodologia epidemiológica. In: Rouquayrol MZ, organizadora. Epidemiologia \& saúde. $6^{\mathrm{a}}$ ed. Rio de Janeiro: MEDSI; 2003. p. 149-78.

14. Ministério da Saúde (BR). Departamento de Informática do Sistema Único de Saúde.[Internet]. Brasília:MS; [acesso em 10 maio 2010]. Cadernos de informações de saúde. Disponível: http://www.datasus.gov.br

15. Minas Gerais. Secretaria de Estado de Saúde. Escola de Saúde Pública do Estado de Minas Gerais. Implantação do Plano Diretor da Atenção Primária à Saúde. Belo Horizonte: ESPMG; 2009.

16. Ministério da Saúde (BR). Política nacional de atenção integral à saúde da mulher: princípios e diretrizes. Brasília: Ministério da Saúde; 2004.

17. Silva DW, Andrade SM, Soares DA, Turini B, Schneck CA, Lopes MLS. Cobertura e fatores associados com a realização do exame Papanicolaou em município do sul do Brasil. Rev Bras Ginecol Obstet. 2006;28(1):24-31.

18. Muller LD, Dias CJS, Luz AMH, Olinto MTA. Cobertura do exame citopatológico do colo do útero na cidade de São Leopoldo, Rio Grande do Sul, Brasil. Cad Saude pública. 2008;24(11):2511-20.

19. Oliveira IR, Inagaki ADM, Daltro AST, Gonçalves LLC, Santos LV. Práticas preventivas e fatores de risco para câncer cervico-uterino entre docentes universitárias. REME Rev Min Enferm. 2009;13(2):238-43.
20. Davim RMB, Torres GV, Silva RAR, Silva DAR. Conhecimento de mulheres de uma unidade básica de saúde da cidade de Natal/RN sobre o exame de Papanicolaou. Rev Esc Enferm USP. 2005;39(3):296-302. 